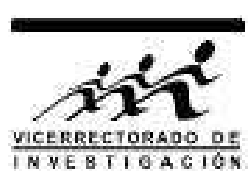

\title{
Uso del OSLO en la enseñanza de la óptica
}

\author{
R. A. Montalvo* y P. H. Rivera \\ Facultad de Ciencias Físicas, Universidad Nacional Mayor de San Marcos, Lima, Perú
}

Recibido 30 diciembre 2012 - Aceptado 30 junio 2013

\begin{abstract}
OSLO es un software que permite diseñar sistemas ópticos en un ambiente gráfico interactivo utilizando un conjunto de elementos ópticos tales como espejos, lentes, prismas, diafragmas, pupilas, mostrando el detalle de los parámetros de cada elemento que se va incorporando sucesivamente, hasta obtener finalmente los parámetros esperados del sistema óptico que se desea construir.

En el presente trabajo, comparamos los modelos teóricos y los resultados del OSLO para el diseño de una lente acromática, un prisma de superficies planas y aplicamos la condición de Scheimpflug para una lente acromática con el objetivo de validar el uso del OSLO como una herramienta computacional para la enseñanza de la óptica geométrica y paraxial.
\end{abstract}

Palabras claves: Diseño óptico, OSLO, óptica geométrica.

\section{Using OSLO for the Optics teaching}

Oslo is a software allow to design optical systems in an interactive graphical environment using an optical elements set such as mirrors, lens, prisms, diafragms, pupils, showing the parameters details of each element which are added succesively to finally obtain the optical system with expected parameters we want to build.

In this paper, we compare the theoretical models and OSLO results for the design of an achromatic len, an parallel plates prism and achromatic len with the Scheimpflug condition for validating purposes of the OSLO as a computer didactic tool for teaching geometric and paraxial optics.

Keywords: Optics design, OSLO, geometrical optics.

Entre los objetivos principales de un curso básico de óptica, el análisis de la formación de las imágenes en distintos dispositivos, la evaluación de las características geométricas de las imágenes y la calidad de las mismas son algunos de los temas que requieren atención especial en la enseñanza de la óptica. La trayectoria de la luz al atravesar un sistema óptico, la localización de los planos principales y focales conformado por varias lentes, así como la obtención de las imágenes intermedias cuando un sistema está conformado por más de una lente, son temas que resultar ser dificultosos en su comprensión para los estudiantes.

Una solución a este problema es el uso de programas que modelan dispositivos ópticos que los estudiantes hayan manipulado alguna vez en su vida diaria tales como los prismáticos, telescopios, proyectores o máquinas fotográficas.

Una herramienta importante que permite diseñar lentes y sistemas ópticos es el Optics Software for Layout and Optimization, OSLO, desarrollado inicialmen- te en la Universidad de Rochester [1. Este es, principalmente, utilizado para determinar las características óptimas de la forma y el tamaño de los elementos ópticos que son utilizados en las cámaras, en los sistemas de comunicaciones ópticas o en las aplicaciones espaciales e instrumentos científicos. Además, permite simular la eficiencia de los sistemas ópticos y el desarrollo de algoritmos requeridos por el usuario para diseños más sofisticados.

El software utiliza la óptica geométrica y paraxial en un interface gráfica donde el usuario puede elegir los dispositivos de una completa base de datos de lentes de distintas empresas comerciales, con diferentes valores de distancia focal, diámetro, espesor y forma. El OSLO facilita la selección del conjunto apropiado de lentes más adecuado para una aplicación específica.

El OSLO EDU versión 6.4.2 Overview [2,3] es gratuito y está destinado para fines educativos con la única limitación que solo permite el modelamiento hasta un máximo de diez superficies ópticas, suministrando un

*rmontalvob@unmsm.com.pe 
gran número de herramientas con muchas posibilidades de uso cuya complejidad puede aumentar conforme se incrementa el número de superfices ópticas.

En el presente trabajo, mostramos dos ejemplos de uso del OSLO, la primera son las simulaciones para la refracción de la luz a través de diferentes medios, y el segundo, el modelamiento y las características de un prisma de placas paralelas; aplicamos también la condición Scheimpflug para una lente acromática y analizamos la aberración cromática para una lente biconvexa. Estas simulaciones son comparados con los cálculos analíticos de la teoría óptica paraxial.

\section{Teoría}

Para el desarrollo de los conceptos que involucra las simulaciones del OSLO, hemos seguido el detalle teórico que se describe en el libro de Smith [4], cuya nomenclatura se adecua más a los términos usados en el OSLO, otros detalles complementarios pueden ser encontrados en la literatura [5-7].

La función de un sistema óptico es colectar una porción de los frentes de onda que proceden de un objeto y por medio de la interposición de cuerpos reflectores y refractores conseguir formar la imagen del objeto, despreciando los efectos de la difracción. El sistema óptico más usado es la lente, que en su forma más general se analiza la incidencia de la luz en una superficie curva en un medio dieléctrico transparente.

El trazado de los rayos, perpendiculares a los frentes de onda, se representan por rayos meridionales que cruzan el eje óptico y los oblícuos que no cruzan el eje óptico pero contribuyen al tamaño y posición de la imagen.

Para calcular la propagación de los rayos a través de los sistemas ópticos, es necesario adoptar una serie de convenciones sobre la nomenclatura y los signos de las variables 4,6. Consideramos el sistema óptico de la Figura 1 que muestra el rayo de luz incidente $\overline{G Q}$ que incide sobre una superficie refractora esférica, la proyección $\overline{G Q P}$ considera el haz incidente sin refracción cruzando el eje óptico a una distancia $L$ de la superficie esférica en el punto $O$. El rayo $\overline{G Q}$ es refractado por la superficie y cruza el eje óptico en $P^{\prime}$, a una distancia $L^{\prime}$ de la superficie. La superficie tiene un radio $R$ con centro de curvatura en $C$ y separa los dos medios de índice de refracción $n$ en el lado izquierdo y $n^{\prime}$ en el derecho. La proyección de la línea $\overline{G Q P}$ hace un (ángulo $\equiv \angle) \angle U$ con el eje óptico para el caso sin refracción, y el rayo $\overline{Q P^{\prime}}$, un $\angle U^{\prime}$ considerando la refracción; $\angle I$ es el ángulo entre el rayo incidente más su proyección lineal $\overline{G Q P}$ y la normal $\overline{H Q C}$ a la superficie refractante que se intersectan en el punto $Q$, y el $\angle I^{\prime}$ está formado entre el rayo refractado $\overline{Q P^{\prime}}$ y la normal $\overline{H Q C}$.
Las convenciones de signos que se observan son 4, a) el radio es positivo si el centro de curvatura está a la derecha de la superficie; b) las distancias a la derecha de la superficie son positivas, a la izquierda, negativas; c) los ángulos de incidencia $\angle I$ y refracción $\angle I^{\prime}$ son positivos si el rayo es rotado en el sentido horario para alcanzar la normal; d) Los ángulos pendientes $\angle U$ y $\angle U^{\prime}$ son positivos si el rayo es rotado en el sentido antihorario para alcanzar al eje óptico. e) La luz viaja de izquierda a derecha.

Un conjunto de ecuaciones se derivan de la Figura 1. por ejemplo en el (triángulo $\equiv \triangle) \triangle P A C$ tenemos que

$$
\overline{C A}=(L-R) \operatorname{sen}(-U)=(R-L) \operatorname{sen} U,
$$

en el $\triangle P^{\prime} A C$ se determina que

$$
\overline{C A^{\prime}}=\left(L^{\prime}-R\right) \operatorname{sen}\left(-U^{\prime}\right),
$$

en el $\triangle Q A C$ observamos que

$$
\operatorname{sen} I=\frac{\overline{C A}}{R},
$$

en el $\triangle Q A^{\prime} C$ se deduce que

$$
\operatorname{sen} I^{\prime}=\frac{\overline{C A^{\prime}}}{R},
$$

$\mathrm{y}$ angularmente se consideran las siguientes relaciones, comenzando en el $\triangle P Q C$, el ángulo exterior $\angle Q C O \equiv$ $-\angle U+\angle I$, para el $\triangle P^{\prime} Q C$ el ángulo exterior $\angle Q C O \equiv$ $\left.-\angle U^{\prime}+\angle I^{\prime}\right)$ y de la igualdad de ambos resultados obtenemos que

$$
\angle U^{\prime}=\angle U-\angle I+\angle I^{\prime} .
$$

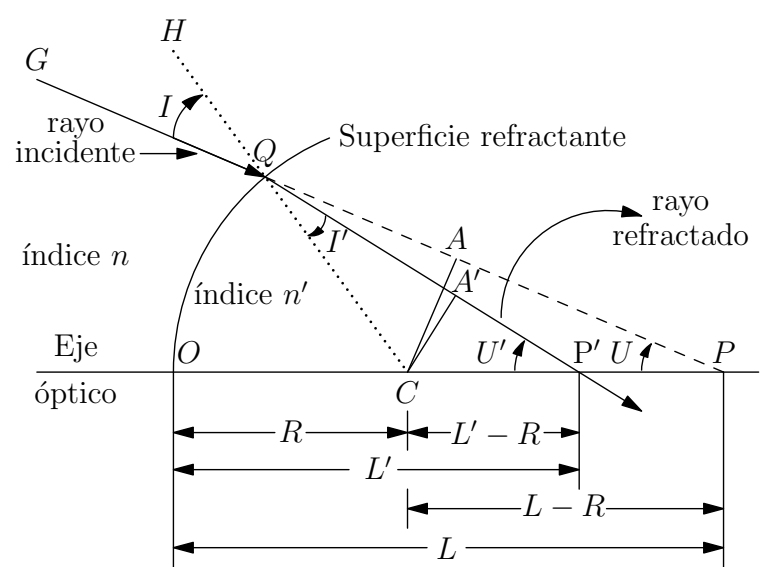

Figura 1: Refracción de la luz por una superficie esférica, esta es muy semejante a las que se presentan en las referencias 4,6,7].

De las relaciones citadas en el párrafo anterior y de la ley de Snell, obtenemos

$$
\overline{C A^{\prime}}=\frac{n}{n^{\prime}} \overline{C A},
$$


donde $n$ y $n^{\prime}$ son los índices de refracción en los medios de la izquierda y la derecha, respectivamente.

En la óptica geométrica se suele usar la aproximación paraxial que consiste en la aproximación de ángulos pequeños, especialmente cuando los rayos de luz van próximos a los ejes ópticos y producen ángulos pequeños con los mismos. A estos rayos se les denominan rayos paraxiales. Si $\theta$ es el ángulo pequeño entre el rayo incidente paraxial y el eje óptico, entonces en primera aproximación tenemos que $\operatorname{sen} \theta \approx \theta, \tan \theta \approx \theta \mathrm{y}$ $\cos \theta \approx 1$. De la misma forma el ángulo de refracción es también pequeño. Para discriminar las ecuaciones de la óptica paraxial de la óptica geométrica usamos las letras minúsculas para la notación paraxial [4, exceptuando el radio de curvatura de la superficie óptica, para ello reescribimos las ecuaciones anteriores basados en la Figura 1 como

$$
\begin{aligned}
\overline{c a} & =(R-l) u, \\
\overline{c a^{\prime}} & =\left(R-l^{\prime}\right) u^{\prime} \Rightarrow l^{\prime}=R-\frac{\overline{c a^{\prime}}}{u^{\prime}}, \\
i & =\frac{\overline{c a}}{R}, \\
i^{\prime} & =\frac{n}{n^{\prime}} i, \\
u^{\prime} & =u-i+i^{\prime} \\
\overline{c a^{\prime}} & =\frac{n}{n^{\prime}} \overline{c a} .
\end{aligned}
$$

Expresando los ángulos en radianes, reemplazamos la Ec.(7) en la Ec.(9) y la ecuación resultante en la Ec.(10). Las últimas dos ecuaciones obtenidas son reemplazadas en la Ec.(11) obteniéndose

$$
\frac{u^{\prime}}{u}=\frac{l}{R}+\frac{n}{n^{\prime}} \frac{(R-l)}{R} .
$$

De la misma forma, reemplazamos las Ecs.(7) y (8) en la Ec.(12) obteniéndose

$$
\frac{u^{\prime}}{u}=\frac{n}{n^{\prime}} \frac{(R-l)}{\left(R-l^{\prime}\right)} .
$$

Igualando estas dos últimas ecuaciones (13) y (14), y realizando un poco de algebra obtenemos

$$
\frac{n^{\prime}}{l^{\prime}}=\frac{\left(n^{\prime}-n\right)}{R}+\frac{n}{l}
$$

que relaciona las distancias $l$ y $l^{\prime}$ de los puntos de intersección entre los haces no refractado y refractado con el eje óptico, respectivamente, los índices de refracción y el radio de curvatura. Esta aproximación se describe como el dominio de primer orden. A la Ec.(15) se le suele llamar como la la ecuación del fabricante de lente.

Considerando que el haz incide sobre la superficie a una altura $y$ respecto al eje óptico entonces los ángulos $u$ y $u^{\prime}$, Fig 1, están dado como $u=y /-l$ y $u^{\prime}=y /-l^{\prime}$, las cuales reemplazamos en la Ec.(15) obteniéndose el parámetro denominado la apertura numérica de la imagen

$$
n^{\prime} u^{\prime}=n u-y \frac{n^{\prime}-n}{R},
$$

donde $n u$ es la apertura numérica del objeto en la aproximación paraxial.

En general, los rayos paraxiales atraviesan más de una superficie refractora, comenzamos analizando el paso de un rayo a través de dos superficies que define un medio óptico tal como se muestra en la Figura 2

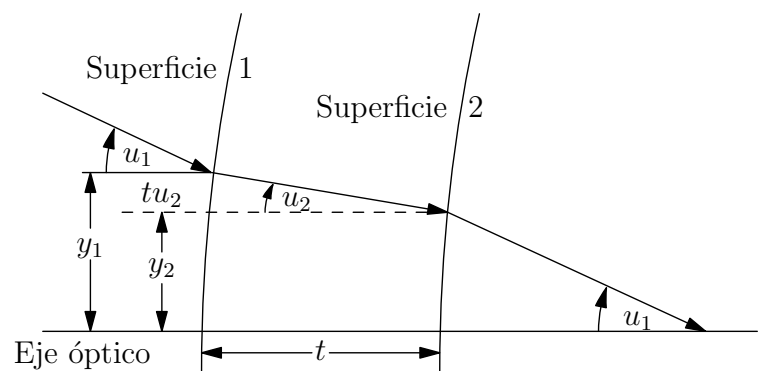

Figura 2: Rayo paraxial atravesando dos superficies separados por un distancia $t, 4$.

El rayo incide en la primera superficie a una altura $y_{1}$ respecto al eje óptico y se refracta a través de la segunda superficie hasta una altura $y_{2}$ respecto al mismo eje. La distancia entre las superficies a lo largo del eje óptico es $t$ y el ángulo de incidencia en el medio óptico sobre la segunda superfice es $u_{2}=u_{1}^{\prime}$ de modo que este haz refractado hace un ángulo $u_{2}^{\prime}$ con el eje óptico. La altura entre el haz incidente y el lado subtendido por el ángulo $u_{1}^{\prime}$ es $t u_{1}^{\prime}$, luego las alturas, considerando la convención considerada líneas arriba, están relacionadas por

$$
y_{2}=y_{1}+t \frac{n_{1}^{\prime} u_{1}^{\prime}}{n_{1}^{\prime}}
$$

y las aperturas numéricas de las superficies debido a la refracción están dadas por

$$
\begin{aligned}
& n_{1}^{\prime} u_{1}^{\prime}=n_{1} u_{1}-y_{1} \frac{\left(n_{1}^{\prime}-n_{1}\right)}{R_{1}} \quad \mathrm{y} \\
& n_{2}^{\prime} u_{2}^{\prime}=n_{2} u_{2}-y_{2} \frac{\left(n_{2}^{\prime}-n_{2}\right)}{R_{2}},
\end{aligned}
$$

donde se observa que $n_{1} u_{1}=n_{2}^{\prime} u_{2}^{\prime}$ si consideramos que el medio que rodea al sistema óptico es el mismo, esto indica que $n_{1}=n_{2}^{\prime}$, luego $u_{1}=u_{2}^{\prime}$ y por otro lado, $n_{1}^{\prime} u_{1}^{\prime}=n_{2} u_{2}$ y $n_{1}^{\prime}=n_{2}$ porque el haz refractado en la primera superficie es el haz incidente en la segunda superficie, luego la apertura numérica está dada por

$$
n_{2}^{\prime} u_{2}^{\prime}=n_{1}^{\prime} u_{1}^{\prime}-y_{2} \frac{\left(n_{2}^{\prime}-n_{1}^{\prime}\right)}{R_{2}} .
$$

Cabe notar que reemplazando la Ec.(18) en la Ec.(20) iniciamos un proceso recursivo donde para una 
superficie $m$ obtenemos que las aperturas numéricas de las imágenes están dadas por

$$
n_{m}^{\prime} u_{m}^{\prime}=n_{m-1}^{\prime} u_{m-1}^{\prime}-\frac{y_{m}\left(n_{m}^{\prime}-n_{m-1}^{\prime}\right)}{R_{m}}
$$

y las alturas recursivamente están dadas por

$$
y_{m}=y_{m-1}+t_{m-1} \frac{n_{m-1}^{\prime} u_{m-1}^{\prime}}{n_{m-1}^{\prime}} .
$$

Estas dos últimas ecuaciones nos permite conocer la apertura numérica de la imagen, la altura de la imagen y su posición respecto a las superficies.

\section{Prisma de planos paralelos}

El prisma es un sistema óptico formado por dos o más superficies planas, no necesariamente pulidas, paralelas entre sí y de cierto espesor $t$, con un índice de refracción $n$ diferente al del aire. El prisma desplaza la posición de la imagen a lo largo del eje óptico invirtiendo la imagen. También se comporta semejante a un diafragma colimando los haces de interés.

En la Figura 3 se aprecia el efecto producido por un prisma de una placa paralela de vidrio, donde $P^{\prime}$ es la distancia donde se forma la imagen desplazada e invertida respecto a la distancia $P$ de la imagen sin prisma. La diferencia de estas dos distancias es $d=P^{\prime}-P$.

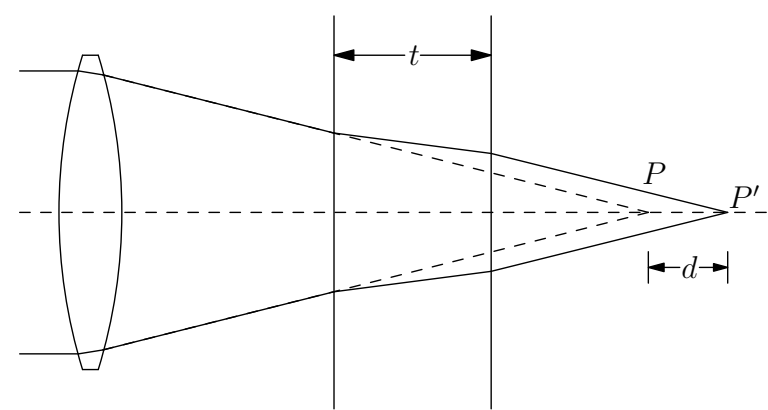

Figura 3: Imagen desplazada por la inserción de un prisma de placa paralela [4].

Para determinar la distancia $d$ en función del índice de refracción del vidrio $n$ y el espesor $t$ de la placa, trazamos la trayectoria de los rayos de luz a través de la placa, Figura 4, y se aprecia que el rayo emergente de la segunda superficie tiene exactamente la misma pendiente que el haz incidente antes de ingresar a la primera superficie, $u_{2}^{\prime}=u_{1}$. Luego, describimos la ley de Snell para las dos superficies en la aproximación paraxial, de la forma

$$
\begin{aligned}
& n_{1} u_{1}=n_{1}^{\prime} u_{1}^{\prime}, \\
& n_{2} u_{2}=n_{2}^{\prime} u_{2}^{\prime},
\end{aligned}
$$

aqui debemos considerar que $n_{1}=n_{2}^{\prime}=n_{a}$ es el índice de refracción del aire, $n_{1}^{\prime}=n_{2}=n$ es el índice de refracción del vidrio, deduciendo que $u_{1}^{\prime}=u_{2} \mathrm{y}$

$$
\frac{u_{2}}{u_{1}}=\frac{n_{1}}{n_{2}} .
$$

En los $\triangle S_{1} O S_{2}$ y $\triangle S_{1} A S_{2}$ observamos que $u_{2}=$ $t / \overline{S_{1} S_{2}}$ y $u_{1}-u_{2}=e / \overline{S_{1} S_{2}}$, respectivamente, de los cuales deducimos que $e=t\left(u_{1}-u_{2}\right)$. Similarmente, en el $\triangle P B P^{\prime}$ observamos que $u_{1}=e / d$, de manera que usando la ecuación previa obtenemos

$$
d=t \frac{n-1}{n}
$$

esta es la distancia en la que se forma la imagen después de introducir un prisma de planos paralelos respecto a la imagen sin prisma en un sistema óptico.

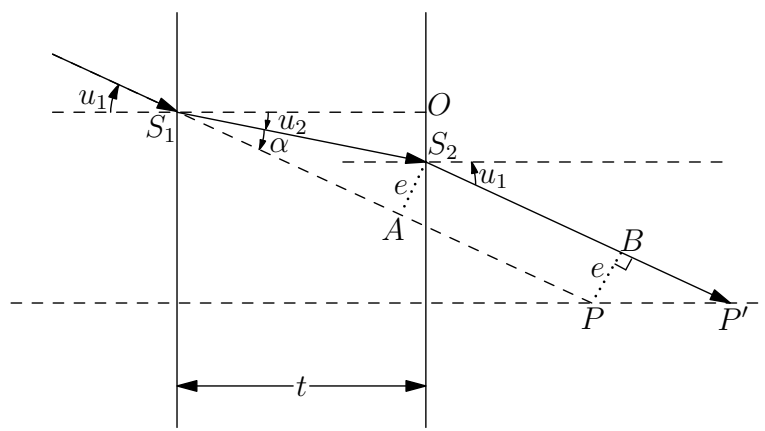

Figura 4: Refracción producida por una placa paralela de vidrio.

\section{La condición de Scheimpflug}

Para una perfecta formación de la imagen de un objeto por una lente, el plano del objeto, los planos de la lente y el plano de la imagen deben ser perpendiculares al eje óptico. Pero no siempre se cumple esta condición, muchas veces el plano del objeto no es paralelo a los planos de la imagen y la lente, y suele observarse que la región próxima al segmento de coincidencia del plano de la imagen con el plano focal posee una buena resolución. Por tanto, un sistema óptico debe ser construído aplicando la regla geométrica que obedece al principio de Scheimpflug, en la que los planos de la lente son proyectados hasta que coinciden con las proyecciones de los planos del objeto, la imagen y el plano focal, a esta línea de coincidencia se le denomina intersección de Scheimpflug [4,7,8,

En la Figura 5, el punto $A=(0,0,0)$ representa el centro del plano objeto y el punto $\mathrm{B}=(0,-y,-z)$ el extremo inferior, respecto a $\mathrm{A}$. El centro del plano de la imagen está en $\mathrm{A}^{\prime}=(0,0,0)$, siendo $\mathrm{B}^{\prime}=(0,-y, z)$ el extremo inferior, respecto a $\mathrm{A}^{\prime}$. La proyección de estos planos se intersectan en el punto $C$ y $D$ con las proyecciones de los planos de la lente. Los puntos $P_{1}$ y $P_{2}$ son los 
puntos principales de la lente, de forma tal que los segmentos definidos $\overline{P_{1} C}$ y $\overline{P_{2} D}$ son obtenidos igualando las tangentes por semejanza de triángulos de modo que

$$
\overline{P_{1} C}=\frac{y}{z} s \quad \text { y } \quad \overline{P_{2} D}=-\frac{y^{\prime}}{z^{\prime}} s^{\prime}
$$

donde $y$ y $z$ son las coordenadas de $B, y^{\prime}$ y $z^{\prime}$ son las coordenadas de $B^{\prime}, s=\overline{P_{1} A}$ es la distancia del objeto y $s^{\prime}=\overline{P_{2} A^{\prime}}$ es la distancia de imagen. Y las relaciones entre los parámetros del objeto y la imagen están dadas por $y^{\prime}=m y, s^{\prime}=m s$ y $z^{\prime}=m^{2} z$ donde $m$ es la magnificación. El principio de Scheimpflug sugiere que $\overline{P_{1} C}=\overline{P_{2} D}$.

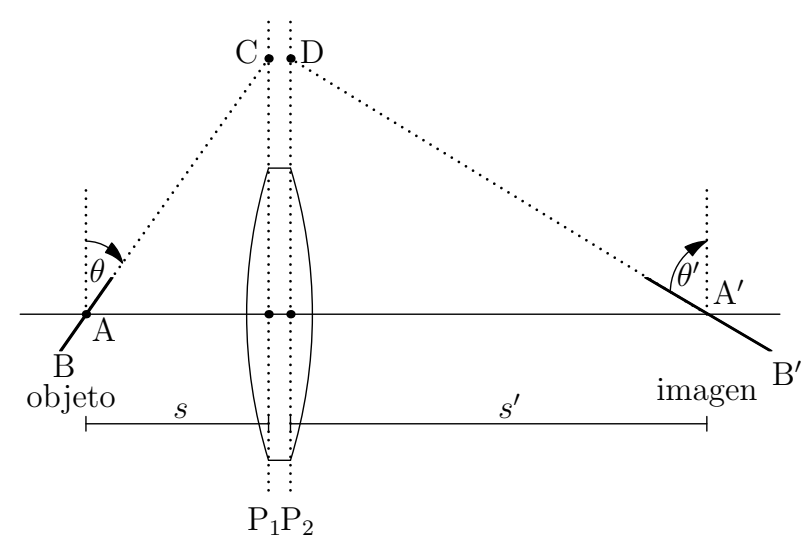

Figura 5: Imagen de un objeto inclinado ilustrando el principio de Scheimpflug [4,7,8].

Este principio es útil para reducir la conocida distorsión trapezoidal presente en las imágenes no paralelas que pierden el fondo. Las intersecciones se encontrarán en el infinito de acuerdo con la condición Scheimpflug por lo que todos los puntos en la plano objeto estarán en el plano del foco independientemente de la abertura y sin perjudicar la resolución de la imagen.

\section{Aberraciones}

Cuando los sistemas ópticos trabajan con aberturas grandes los rayos marginales focalizan en un punto diferente al punto de focalización de los rayos paraxiales y generan alteraciones en la forma y color de las imágenes a las cuales se les denomina aberraciones.

Las aberraciones fueron estudiadas por primera vez por Seidel 7 , 9], quién en un estudio sistemático determinó que las aberraciones que se manifiestan debido a la quiebra de simetría axial de las variables ópticas al construir la imagen respecto al eje óptico son la esférica, el coma, el astigmatismo, la curvatura Petzval y la distorsión. A estas aberraciones de les denomina las aberraciones de Seidel que suelen ser de tercer, quinto y séptimo orden [4, 7, 9].
Por otro lado, la aberración cromática se produce debido a la variación del índice de refracción con la longitud de onda; como consecuencia las propiedades de los elementos ópticos dependen de las diferentes longitudes de onda. La aberración cromática axial se manifiesta como una variación longitudinal formando un pequeño espectro alrededor del foco de la lente y la aberración cromática lateral se manifiesta produciendo diferentes aumentos para cada longitud de onda afectando los detalles tangenciales de la imagen.

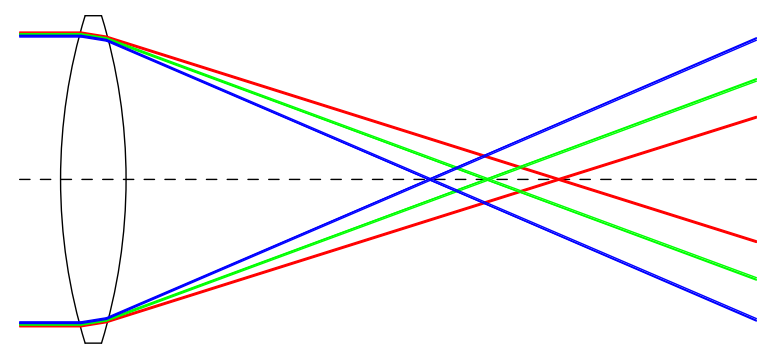

Figura 6: Diferentes longitudes de onda al atravesar el sistema óptico generan diferentes planos de imagen debido a que el índice de refracción de una lente depende de la longitud de onda [10].

\section{Resultados}

El OSLO presenta una interface de usuario con tres ventanas; la primera corresponde al editor de los parámetros de entrada de los elementos ópticos (Surface Data), la segunda a la salida de los parámetros calculados y el detalle de los parámetros de cada elemento óptico de acuerdo a la base de datos del OSLO (TW) y la tercera a la ventana donde se muestran las diferentes figuras de los diferentes parámetros de salida (UW) [10].

\section{Refracción en diferentes superficies}

El primer caso que presentamos es la formación de la imagen producida por una lente acromática, formada por tres superficies con los radios de curvaturas dados por $R_{1}=+50 \mathrm{~mm}, R_{2}=-50 \mathrm{~mm}$ y $R_{3}=\infty$. Haciendo un análisis detallado del proceso de la formación de la imagen, explicamos el detalle de la imagen formada por la primera superficie para el cual los datos iniciales son: a) la distancia del objeto a la superfice $1, s_{1}=-300$ $\mathrm{mm}, b$ ) la altura del objeto $\left.y_{1}=20 \mathrm{~mm}, c\right)$ el índice de refracción del medio (aire) antes de la superficie 1 , $n=1, d$ ) el índice de refracción del medio (vidrio K10) después de la superficie $1, n_{1}=1.50137$, e) el radio de la apertura del cono de los rayos que inciden sobre la superficie es $\operatorname{rap}=10 \mathrm{~mm}$.

El OSLO desarrolla las ecuaciones de diseño en forma exacta considerando las aperturas numéricas del objeto y la imagen como $n \operatorname{sen} u$ y $n^{\prime} \operatorname{sen} u^{\prime}$, respectivamen- 
te. Esto se aprecia en el Setup de la ventana Surface Data que calcula los parámetros de salida a partir de los datos de entrada. También posee otro módulo que proporciona los resultados considerando la aproximación paraxial y que se muestran en la ventana de resultados (TW), en la opción de Aberrations.

Los datos de salida que proporciona el Setup indica que los rayos inciden con un ángulo $\theta=0.033315 \mathrm{rad}$ asociado a la apertura numérica del objeto que es igual a $n \operatorname{sen} \theta$ donde $\theta$ es la mitad del ángulo del cono de luz, la pendiente del rayo axial -el ángulo entre el rayo axial y el eje paraxial paralelo al eje óptico-, es -0.047827 rad luego de atravesar la última superficie; la apertura numérica de la imagen, $n_{1} \operatorname{sen} \theta_{1}$-donde $\theta_{1}$ es el ángulo del cono de luz en el espacio imagen-, es 0.047800. La altura final de la imagen es $-13.939143 \mathrm{~mm}$, a una distancia de $198.515781 \mathrm{~mm}$ con una distancia focal efectiva de $122.542337 \mathrm{~mm}$.

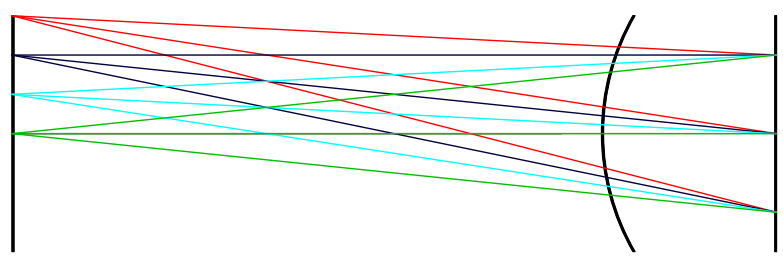

Figura 7: Trazado de los rayos para la primera superficie explicada en el texto donde la imagen es formada a una distancia de $10 \mathrm{~mm}$ de la superficie, valor dado para una mejor visualización del trazado de los rayos.

En la aproximación paraxial, los resultados que muestra el OSLO en Aberrations en la ventana de resultados $T W$ nos proporciona información específica para cada superficie del dispositivo óptico. Analizamos la primera superficie considerando que el radio del cono de entrada de luz es $10 \mathrm{~mm}$, produciendo una apertura numérica del objeto de $\theta=0.033333 \mathrm{rad}$ con una pendiente del rayo axial de -0.044586 . La pendiente del rayo principal es $-0.044404 \mathrm{rad}$ y la pendiente del rayo principal después de la superficie es igual a -0.066667 rad, ver Figura 7

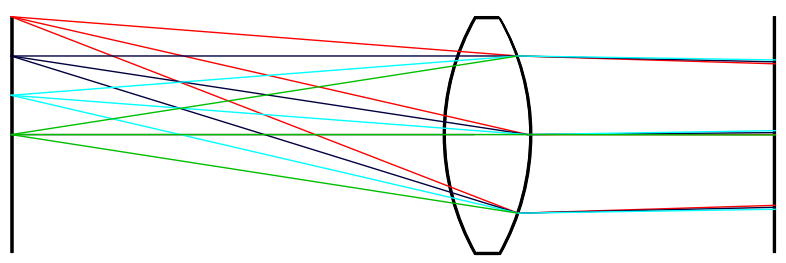

Figura 8: Trazado de los rayos para la primera y segunda superficie con una imagen formada a $10 \mathrm{~mm}$ para una mejor visualización de los rayos.

Se adiciona la segunda superficie a $10 \mathrm{~mm}$ de la primera, ambas separan un medio de índice de refracción $n_{1}=1.50137$, el radio del cono de luz que parte de esta segunda superficie es $9.554135 \mathrm{~mm}$, la pendiente del rayo axial con la que ingresa a esta segunda superficie es -0.029866 y la pendiente del rayo principal es -0.042185; este rayo principal al pasar por el foco se desvía del eje óptico e se intersecta con la segunda superficie a una altura de $-0.444039 \mathrm{~mm}$; al salir de esta segunda superficie la pendiente del rayo principal es -0.035523 , Figura 8.

Finalmente se agrega la tercera y última superficie a $2 \mathrm{~mm}$ de distancia respecto a la segunda separando un medio con un índice de refracción $n_{2}=1.601401$. El radio del cono de entrada del haz es $9.494404 \mathrm{~mm}$ cuya pendiente del rayo axial es -0.047827 y la pendiente del rayo principal -0.067555 , este rayo principal al pasar por el foco se desvía del eje óptico e intersecta la tercera superficie a una altura de $-0.528408 \mathrm{~mm}$; al salir de esta tercera superficie la pendiente del rayo principal es -0.042185. La imagen final formada es de -13.939143 mm y la apertura numérica de la imagen igual a $-0.067555 \mathrm{rad}$, Figura 9

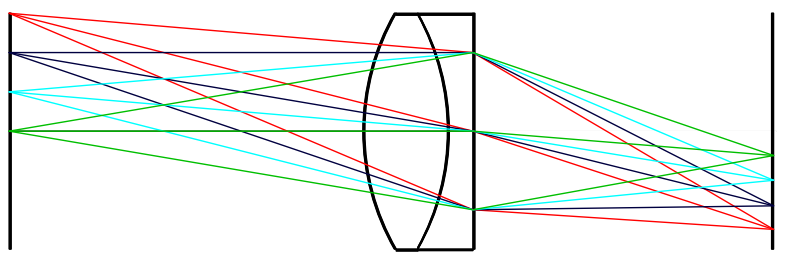

Figura 9: Se muestra el trazado de los rayos para tres superficies que conforman una lente acromática. El detalle del trazado de los rayos para cada superficie se muestran en el texto.

Ahora comparamos estos resultados del OSLO, de aberrations en la ventana $T W$, con los datos que nos proporciona el modelo teórico en la aproximación paraxial discutido en la sección de la teoría; los rayos al incidir en la primera superficie lo hacen con una apertura numérica del objeto que es igual a $n \operatorname{sen} \theta$, -donde $\theta$ es la mitad del ángulo del cono-, que en la aproximación paraxial es igual a $n \theta$. De la geometría se obtiene que $\theta \approx \mathrm{rap} / \sqrt{s^{2}+\mathrm{rap}^{2}}=0.033315$.

La apertura numérica de la imagen se obtiene de la Ec.(16) resultando $n_{1}^{\prime} u_{1}^{\prime}=-0.066959 \mathrm{rad}$, con una diferencia de $2.9 \times 10^{-4} \mathrm{~mm}$ respecto al resultado del OSLO, -0.066666. La altura de la imagen producida por la refracción en el medio que separa la primera y segunda superficies está dado por la Ec.(17) resultando $y_{2}=9.554705 \mathrm{~mm}$, el OSLO nos brinda una altura de $9.554135 \mathrm{~mm}$ por lo que la diferencia es $5.7 \times 10^{-4} \mathrm{~mm}$.

Añadimos la segunda superficie y como $n_{2} u_{2}=$ $n_{1}^{\prime} u_{1}^{\prime}$, debido a que el haz refractado de la primera superficie es el incidente de la segunda, la apertura numérica de la imagen cuando el haz atraviesa la segunda superficie, Ec.(19), es $n_{2}^{\prime} u_{2}^{\prime}=-0.048289 \mathrm{rad}$. Este ángulo refractado es muy próximo al determinado por el 
OSLO, $0.047800 \mathrm{rad}$, con una diferencia entre ambos de $4.9 \times 10^{-4} \mathrm{rad}$.

$\mathrm{Al}$ incorporar la tercera superficie, la altura de la imagen calculada por la Ec.(22) resulta $y_{3}=9.494397$ mm. En el OSLO el resultado es de $9.494404 \mathrm{~mm}$ y la diferencia entre ambos resultados es $7 \times 10^{-6} \mathrm{~mm}$. Como la última superficie del sistema óptico es plano, su radio de curvatura es cero por lo tanto la apertura numérica es, $n_{3}^{\prime} u_{3}^{\prime}=-0.048289 \mathrm{rad}$.

La ubicación de la imagen final del sistema óptico, se determina a partir de $y_{3}=n_{3}^{\prime} u_{3}^{\prime} \times s_{3}^{\prime}$, donde $s_{3}^{\prime}=196.616144 \mathrm{~mm}$, el OSLO produce 198.515781 $\mathrm{mm}$, es decir, una diferencia entre ambos resultados de $1.899637 \mathrm{~mm} \approx 0.96 \%$.

La determinación de la altura de la imagen real formada por el sistema óptico se basa en la Ec.(22), el OSLO determina la misma considerando que el ángulo de incidencia para la aproximación paraxial es $n_{1} u_{1}=-0.066667$ rad, Figura 10] La refracción aleja el haz principal del eje óptico de modo que alcanza una altura de $y_{2}=-0.444034 \mathrm{~mm}$ al incidir en la segunda superficie luego de recorrer una distancia de $10 \mathrm{~mm}$ en un medio con un índice $n_{1}^{\prime}=1.50137$ que separa ambas superficies. El OSLO calcula una altura $y_{2}=-0.444039$ $\mathrm{mm}$, estimando una diferencia de $5 \times 10^{-6} \mathrm{~mm}$.

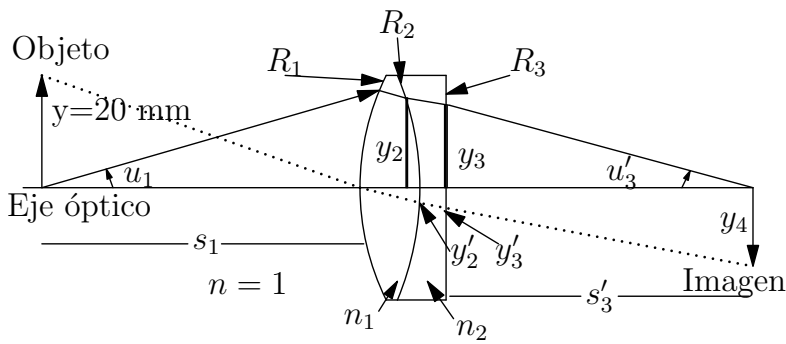

Figura 10: Se muestra el trazado de los rayos para las tres superficies que conforman una lente acromática.

Cuando el rayo principal alcanza la tercera superficie posee una pendiente de $n_{2}^{\prime} u_{2}^{\prime}=-0.065949 \mathrm{rad}$, alcanzando una altura $y_{3}=-0.526398 \mathrm{~mm}$, mientras que, el OSLO produce $y_{3}=-0.528408 \mathrm{~mm}$, considerando una diferencia de $2 \times 10^{-3} \mathrm{~mm}$.

Finalmente, la altura de la imagen formada por la lente acromática descrita es determinada por la Ec.(22), $y_{3}=-13.808801 \mathrm{~mm}$, en el programa OSLO el cálculo de la altura de la imagen es $13.939143 \mathrm{~mm}$; lo que la diferencia de nuestro calculo en $0.130342 \mathrm{~mm} \approx 0.94 \%$.

\section{Prisma de placas paralelas}

A continuación presentamos los resultados del modelamiento de un prisma de placas paralelas en el OSLO. En primer lugar, determinamos la formación de la imagen con una lente acromática, la cual está conformada por tres superficies cuyos radios de curvatura son
$R_{1}=+32.29 \mathrm{~mm}, R_{2}=-25.43 \mathrm{~mm} \mathrm{y} R_{3}=-81.06$ $\mathrm{mm}$, con una distancia focal efectiva igual a $50 \mathrm{~mm}$. Los datos de entrada en la ventana Surface Data para determinar la formación de la imagen producida por la lente acromática son: $a$ ) la distancia del objeto a la superfice $\left.1, s_{1}=-1500 \mathrm{~mm}, b\right)$ la altura del objeto $\left.y_{1}=50 \mathrm{~mm}, c\right)$ el índice de refracción $n=1$ del medio (aire) antes de la superficie $1, d$ ) el índice de refracción $n_{1}=1.56883$ del medio (vidrio N-BAK4) después de la superficie 1,e) el espesor entre la primera y segunda superficie, $t_{12}=4.83 \mathrm{~mm}, f$ ) el índice de refracción $n_{2}=1.72828$ del medio (vidrio N-SF10) después de la superficie $2, g$ ) espesor entre la segunda y tercera superficie, $\left.t_{23}=3.00 \mathrm{~mm}, h\right)$ y el radio de la apertura del cono de los rayos que inciden sobre la superficie es $a p=10 \mathrm{~mm}$.

Los cálculos realizados por el OSLO se encuentran en la opción Setup de la ventana Surface Data donde se puede observar que el plano de la imagen se ubica a una distancia de $47.994084 \mathrm{~mm}$, para un tamaño de imagen de $1.722605 \mathrm{~mm}$ y una longitud focal efectiva de $49.997415 \mathrm{~mm}$.

En la Figura 11 se aprecia la distancia calculada por el programa desde la última superficie de la lente acromática hasta el plano imagen en el punto $P=47.994084 \mathrm{~mm}$.

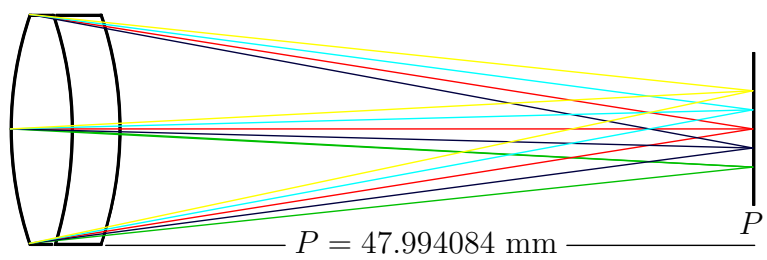

Figura 11: Trazado de los rayos de la lente acromática hasta el plano imagen. El detalle muestra solo la distancia de la última superficie de la lente hasta el plano imagen para una mejor visualización de los rayos.

En segundo lugar, añadimos el prisma que está formado por dos placas paralelas de vidrio. En la misma ventana, Surface Data, usada para la lente acromática se adiciona los datos del prisma, los cuales son: a) dos placas de vidrio BK7 cuyo índice de refracción es $\left.n_{3}=1.51680, b\right)$ el espesor de cada placa es $t=2$ $\mathrm{mm}, c)$ y la distancia de separación entre las placas 3 $\mathrm{mm}$.

Los cálculos realizados por el OSLO con ambos elementos, la lente acromática y el prisma de placas paralelas, ubica el plano de la imagen a una distancia igual a $39.356954 \mathrm{~mm}$, para un tamaño de imagen de 1.722605 $\mathrm{mm}$ y una longitud focal efectiva de $49.997415 \mathrm{~mm}$. La Figura 12 muestra la distancia a la cual se forma la imagen apartir de la última superficie de la placa paralela, la cual es $39.356954 \mathrm{~mm}$. En la misma figura se aprecia que la distancia a partir de la última superficie de 
la lente acromática hasta la imagen en $P^{\prime}$ es 49.356954 $\mathrm{mm}$.

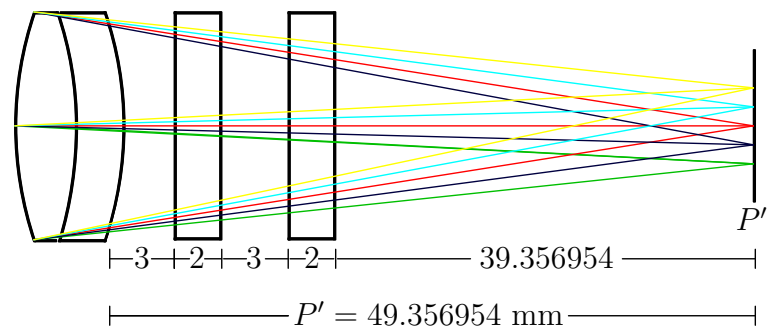

Figura 12: Trazado de los rayos de la lente acromática y el prisma de placas paralelas hasta el plano imagen. El detalle muestra la distancia de la última superficie de la lente hasta el plano y las distancias entre los elementos ópticos para una mejor visualización. Los números están en mm.

Tomando los valores calculados por el OSLO, el desplazamiento de la imagen debido a la insercion del prisma de placas paralelas es $d=P^{\prime}-P=1.362870 \mathrm{~mm}$. El resultado que produce la Ec.(26), para una placa es $0.6814345 \mathrm{~mm}$ y para dos placas el desplazamiento total es $d=1.362870 \mathrm{~mm}$, comparando ambos resultados no se aprecia diferencias significativas.

\section{La condición de Scheimpflug}

Cuando el plano del objeto no es paralelo a los planos de la imagen y de la lente, la distorsión que se aprecia en la Figura 13 es el efecto denominado el ojo de cerradura debido a la distorsión trapezoidal que se forma en un plano inclinado respecto al plano de la imagen, como suele ocurrir con un proyector. La distorsión de las dimensiones de la imagen hace que se vea como un trapecio.

En el caso típico de un proyector el objetivo enfoca a una distancia media y no posee la suficiente profundidad de foco necesaria para mantener todos los haces en un plano perpendicular al eje óptico luego produce una imagen más grande en la parte inferior que en la parte superior.

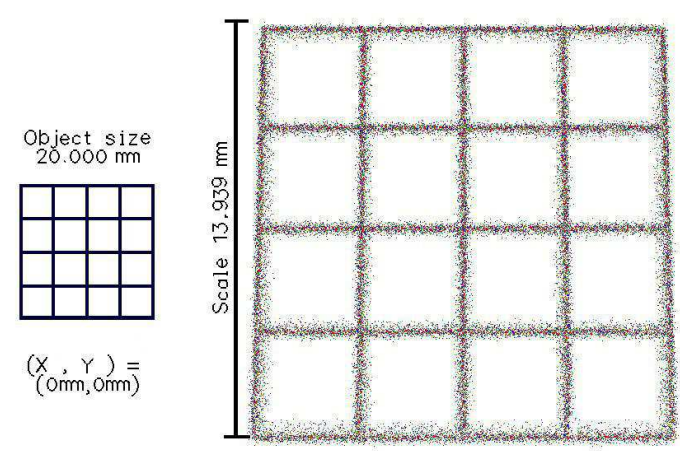

Figura 13: Se muestra el efecto del ojo de cerradura.
Por otro lado, usando la lente acromática de la sección anterior, le aplicamos un giro de $20^{\circ}$ al objeto ubicado delante de la lente, la formación de la imagen ocurre en un plano inclinado que el OSLO determina considerando la condición de Scheimpflug, Figura 14.

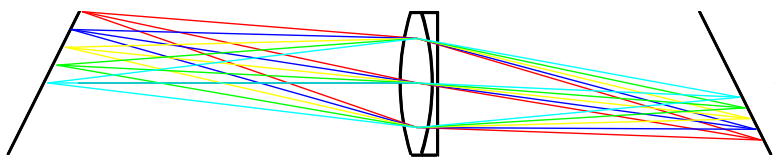

Figura 14: Imagen formada debido a un objeto inclinado.

En la Figura 15. se muestra la comparación de las profundidades de foco de una lente acromática con otra lente acromática bajo la condición de Scheimpflug. Siendo $\delta$ la profundidad de foco lente acromático con la condición de Schempflug y $\delta^{\prime}$ la profundidad de foco de la lente acromática. Se observa que $\delta>\delta^{\prime}$

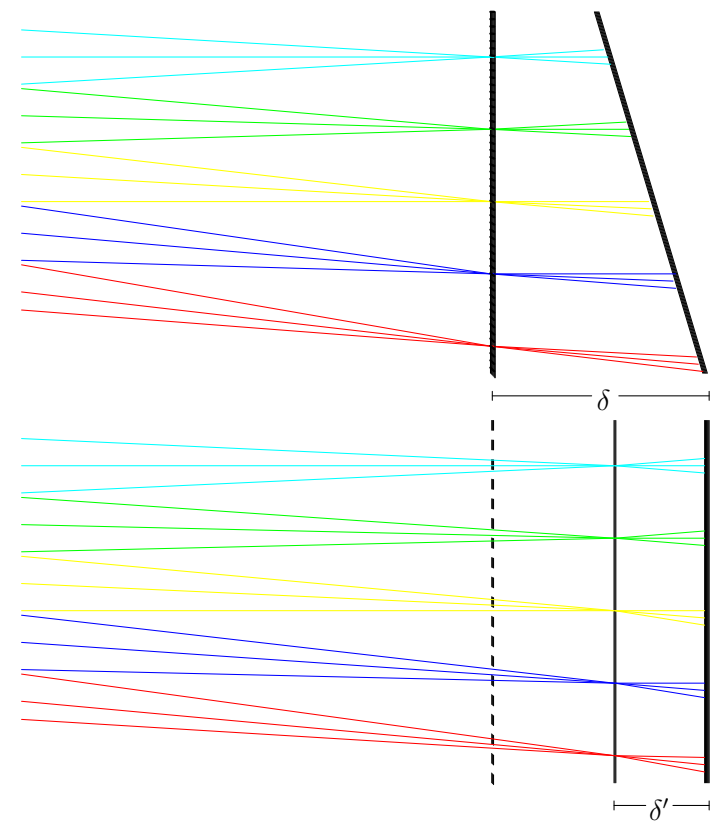

Figura 15: Variación de la profundidad de foco con y sin la condición de Scheimpflug.

\section{La aberración cromática}

La determinación de la aberración cromática con el OSLO fue realizada con la lente biconvexa, la cual está conformada por dos superficies cuyos radios de curvatura son $R_{1}=+32.29 \mathrm{~mm} \mathrm{y} R_{2}=-25.43 \mathrm{~mm}$, con una distancia focal efectiva igual a $100 \mathrm{~mm}$. Los datos de entrada en la ventana Surface Data para la lente biconvexa son: a) la distancia del objeto a la superfice $\left.1, s_{1}=-1500 \mathrm{~mm} ; b\right)$ la altura del objeto $y_{1}=50$ $\mathrm{mm} ; c$ ) el índice de refracción $n=1$ del medio (aire) antes de la superficie $1 ; d$ ) el índice de refracción 
$n_{1}=1.56883$ del medio (vidrio N-BAK4) después de la superficie $1 ; e)$ el espesor entre la primera y segunda superficie, $t_{12}=4.83 \mathrm{~mm}$; f) el índice de refracción $n_{2}=1.60568$ del medio (vidrio N-BAF4) al ingresar a la superficie $2 ; g$ ) y el radio de la apertura del cono de los rayos que inciden sobre la superficie es $a p=10 \mathrm{~mm}$, Figura 16.

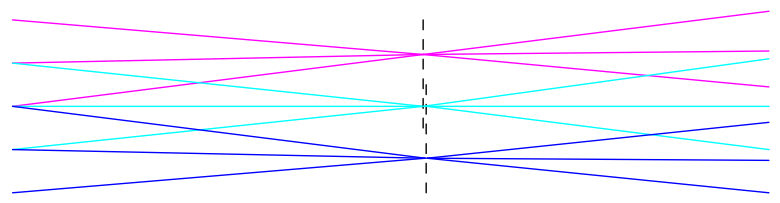

Figura 16: Detalle de los puntos focales calculados por el OSLO para diferentes longitudes de onda.

Los resultados proporcionados por el OSLO, en la subventana aberrations en la ventana $T W$, son 0.017396 para el color lateral primario y para el color lateral secundario es -0.012208. La Figura 17 muestra el ploteo del color lateral primario y secundario para los intervalos de longitud de onda de 0.48618 a 0.58756 $\mu \mathrm{m}$ y 0.58756 a $0.65627 \mu \mathrm{m}$.

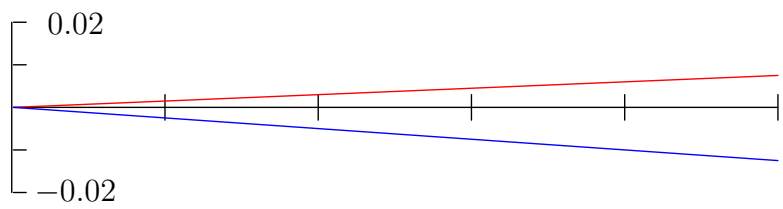

Figura 17: Aberración para la longitud de onda primaria y secundaria versus la fracción de la altura del objeto.

\section{Conclusiones}

El OSLO es una buena herramienta para analizar el detalle de cálculo de los diferentes parámetros que resultan de introducir elementos ópticos para el diseño de un sistema óptico para una utilidad definida. Permite visualizar la evolución del sistema en tiempo real al modificar las variables de diseño lo que permite comprender mejor el funcionamiento de los distintos instrumentos ópticos y la formación de las imágenes mediante el diagrama de los rayos para distintas situaciones planteadas. En este trabajo se han presentado los diagramas $2 \mathrm{D}$, pero también permite obtener los procesos en 3D.

La versión educativa del OSLO es usada para el estudio de la óptica básica y el diseño de algunos instrumentos básicos que permitan visualizar el detalle de la aplicación de los modelos de la óptica geométrica y paraxial.

Los casos modelados con el OSLO han sido verificados con sus respectivos cálculos analíticos, en el primer caso, el análisis de la refracción para diferentes superficies que separan medios diferentes y en el segundo caso, el prisma de un sistema óptico cuyos los valores finales del corrimiento de las imágenes casi no difieren. Adicionalmente hemos realizado una evaluación gráfica cualitativa, como es la condición de Scheimpflug y la aberración cromática confirmando el modelo desarrollado en la óptica.

Existen otros programas de diseño óptico, comerciales y no comerciales, pero el OSLO a pesar de su complejidad en los detalles de diseño, muestra un óptimo desempeño en la formación de imágenes, por tanto, creemos que el OSLO puede ser de gran ayuda para que los estudiantes mejoren su comprensión de los procesos de la formación de imágenes en los diferentes dispositivos ópticos.

\section{Referencias}

[1] $\mathrm{Su}$ Geun Chae, OSLO Optical design software as an educational tool, Portland State University. sugeun@pdx.edu, www.genuineholographics.com/oslo.html

[2] en.wikipedia.org/wiki/Optics_Software_for_ Layout_and_Optimization

[3] lambdares.com/products/oslo/index.html

[4] Warren J. Smith, Modern Optical Engineering, cuarta edición, Mac Graw Hill, New York (2008).

[5] Eugene Hecht, Optics, cuarta edición, Addison Wesley, New York (2002).
[6] Javier Alda, Paraxial Optics, Encyclopedia of Optical Engineering, pp. 1920-1931, Eds. Marcel y Dekker, Londres (2003).

[7] Rudolf Kingslake y R. Barry Johnson, Lens Design Fundamentals, segunda edición, Academic Press, Elsevier, Amsterdam (2010).

[8] http://en.wikipedia.org/wiki/Scheimpflug_ principle

[9] http://en.wikipedia.org/wiki/Chromatic_ aberration

[10] Bogdan Nedić, Darko Vasiljević, Nataša Vesić, Complexity of Designing Optical Systems, (2007) www.genuineholographics.com/oslo.html 